\title{
Factores situacionales que influyen en el aprendizaje de la taxonomía NOC en alumnos de enfermería
}

\section{Situational factors affecting the learning of the taxonomy noc nursing students}

Lic. en Enf. Adiel Agama Sarabia.

Dra. Silvia Crespo Knopfler.•

Dra. Susana González Velázquez•.•

\section{Resumen}

Introducción. En el caso de la enseñanza en enfermería en la FES Zaragoza, esta implementa la utilización de la taxonomía Noc, por lo cual identificar cuáles son los factores que influyen en su aprendizaje, le permitirá al docente incrementar la significancia de su enseñanza. Objetivos: Identificar los factores situacionales que influyen en el aprendizaje de la taxonomía noc en alumnos de Enfermería y establecer la relación que existe entre estos factores y el aprendizaje de la taxonomía. Metodología: Estudio transversal y correlacional, con un muestreo estratificado por conglomerados, con un total de 224 alumnos; mediante un instrumento, con un alfa de Cronbach de 0.984 , que midió la percepción del alumno sobre los factores situacionales que influyen en su aprendizaje,. El análisis incluyó medidas de tendencia central y pruebas de correlación de Pearson. Resultados: La dimensión mejor evaluada fue "Las relaciones interpersonales" con el 89.4\% de los alumnos que lo calificó como adecuado. La dimensión "Habilidades Docentes", obtuvo un valor de $r_{p}=0.939$ y un grado sig. 0.000 por lo cual se puede decir que tiene una relación positiva muy fuerte. Discusión: La literatura refiere a las "Relaciones Interpersonales" como uno de los factores principales en el aprendizaje; a diferencia del presente estudio donde se identificô a las "Habilidades Docentes" como factor principal. Conclusiones: Las habilidades docentes es el factor que más influye para el aprendizaje de la taxonomía NOc; conjuntamente con una adecuada retroalimentación de los aprendizajes, se puede incrementar la significancia en el aprendizaje que tiene para el alumno de enfermería.

- Licenciado en Enfermería. Colaborador de la Línea de Investigación en Evaluación Institucional FES-Zaragoza, UNAM. ..Profesor de Carrera Titular "B" Facultad de Estudios Superiores Zaragoza. UNAM.

... Profesor de Carrera Titular "A” Facultad de Estudios Superiores Zaragoza, UNAM.

Correo Electrónico. Lic. en Enf. Adiel Agama Sarabia: agamael_22@hotmail.com

FeCha de ReCibido: 1 DE SePtiembre 2010

Fecha de EnViado: 7 DE SePtiembre 2010

ACeptado: 28 DE Septiembre 2010

Factores Situacionales,

Taxonomía NOC,

Aprendizaje 
Introduction: The results are encouraging assurance programs and quality improvement. In the case of nursing education in the FES Zaragoza, this implements the use of Noc taxonomy, and therefore identify the factors that influence their learning, will allow the teacher to increase the significance of his teaching. Objectives: To identify the situational factors that influence learning Noc taxonomy in nursing students and establish the relationship between these factors and learning taxonomy. Methodology: A cross-sectional and correlational, with a stratified cluster sampling, with a total of 224 students in third and fourth year of the race by an instrument of 53 items, with a Cronbach alpha of 0.984 , which measured student perception on situational factors that influence their learning. The analysis included measures of central tendency and Pearson correlation tests. Results: Predominantly female with $83.5 \%$. The best dimension evaluated was "interpersonal relations" with $89.4 \%$ of students who called it right. Dimension "Teaching Skills", obtained a value of $r_{p}=0.939$ and an actual sig. 0.000 so it can be said to have a very strong positive relationship. Discussion: The literature refers to "interpersonal relations" as one of the key factors in learning, unlike the present study which identified the "Teaching Skills" as a major factor. Conclusions: The teaching skills is the main factor for learning Noc taxonomy, together with adequate feedback of learning, can increase the significance it has for the nursing student.

\section{INTRODUCCIÓN}

Las instituciones, como las enfermeras profesionales convergen en que la calidad del cuidado enfermero se enfoca en los resultados, utilizando entre otros los indicadores propuestos por la Nursing Outcomes Classification (NOC). En este sentido los resultados son el estímulo de los programas de garantía o mejora de calidad en la atención de los pacientes. En el caso de la enseñanza de la carrera de enfermería en la FEs Zaragoza, ésta implementa la utilización de la taxonomía NOC, por lo cual identificar cuáles son las variables que influyen en su aprendizaje, le permitirá al docente incrementar la significancia de su enseñanza.

La complejidad que implica el incluir las diferentes variables que influyen en el proceso de aprendizaje, y no sólo por el hecho de su simple inclusión, si no por las inte- rrelaciones que se dan entre ellas, aunado a las dificultades naturales de cuantificar su presencia y sus efectos en todo el proceso educativo. Sin embargo, al caracterizarlas se han separado en dos categorías:

a) La categoría cognitiva o variables intrapersonales y $b$ ) la categoría de variables de tipo afectivo-social. Esta segunda categoría considera los factores afectivos y sociales del aprendizaje o factores situacionales dentro de las cuales se incluyen entre otros: variables motivacionales y actitudinales, factores sociales y de grupo, la naturaleza del aula y características del profesor. $^{1}$

Diversos autores, entre ellos Díaz Barriga-Arceo, ${ }^{2}$ han postulado que es mediante la realización de aprendizajes significativos, que la construcción del conocimiento escolar es en realidad un proceso de elaboración, en el sentido de que el alumno selecciona, organiza y transforma la información que recibe de muy diversas fuentes, estableciendo relaciones entre dicha información y sus ideas o conocimientos previos. Así, aprender un contenido quiere decir que el alumno le atribuye un significado, construye una representación mental a través de imágenes o proposiciones verbales, y reconstruye uno nuevo como marco explicativo de dicho conocimiento. ${ }^{3}$

De acuerdo con Ausubel, durante el aprendizaje significativo el aprendiz relaciona de manera sustancial la nueva información con sus conocimientos y experiencias previas. ${ }^{4}$ Por otro lado, también importa la forma en que se plantean los materiales de estudio y las experiencias educativas. Si se logra el aprendizaje significativo, se tras- 
ciende la repetición memorística de contenidos inconexos y se logra construir significado, dar sentido a lo aprendido y entender su ámbito de aplicación y relevancia en situaciones académicas y cotidianas.

De esta manera los tres aspectos clave que deben favorecer este proceso instruccional serán el logro del aprendizaje significativo, la memorización comprensiva de los contenidos escolares y la funcionalidad de lo aprendido. ${ }^{5}$

El tratar de integrar los diferentes factores que influyen en el proceso de aprendizaje implica una gran complejidad, sobre todo cuando se pretende lograr que sea significativo para el estudiante, no obstante, se toman los principales factores mencionados por Díaz Barriga ${ }^{6}$ para lograr un aprendizaje significativo, los cuales a su vez corresponden los factores cognitivos y situacionales, siendo estos últimos los que Ausubel, Novak y Hanesian, consideran de gran influencia para el aprendizaje. Estos factores pueden subdividirse a su vez en:

\section{El docente}

El docente constituye una de las variables que más influye en el proceso de enseñanza y aprendizaje, tanto por el grado de conocimiento de la materia que imparte, como por su estilo para presentar y organizar el material de aprendizaje, o bien, por su capacidad para comunicarse y transmitir valores a los alumnos. ${ }^{7}$

\section{Factores sociales en el aprendizaje}

Las variables sociales y de grupo deben ser consideradas en el aprendizaje escolar, pues inciden inevitablemente en el aprendizaje de la materia de estudio. Aunque el peso de la evidencia indica que el clima del salón de clase tiene poco efecto en el aprovechamiento académico, afecta las actitudes hacia la escuela, el aprendizaje de valores culturalmente aprobados y la conducta escolar. $^{8}$

\section{El contexto del aula}

El aula es un sistema de actividad humana, donde elementos humanos y materiales se encuentran en continua relación mediante un flujo constante de información que le confieren una determinada organización característica, en el cual el pensamiento y la práctica de profesores y alumnos están mediatizados y condicionados, por el intercambio que se establece en él, entre las conductas y actuaciones de los alumnos por un lado y la evaluación del profesor por otro. Esta dimensión evaluadora se presenta como mediadora en la comunicación, manifestándose en una estructura de poder característica donde el profesor regula y gobierna el flujo de esa comunicación.

Por su parte, los alumnos en clase se encuentran sometidos a una dimensión evaluadora y disciplinar donde no sólo ellos, sino toda la escuela es la que se evalúa. Los profesores y estudiantes no actúan, piensan o se relacionan desde una estructura igualitaria, sino que lo hacen desde una situación jerárquica y de poder. ${ }^{9}$

Por lo tanto queda claro que los profesores necesitan saber cómo opera la comprensión de los estudiantes para diseñar estrategias de enseñanza que posibiliten mejorar la eficiencia de los procesos. Sin embargo, ello no se aplica a la realidad que todos los docentes segu- ramente reconocen, esto es, que los alumnos no transfieren su conocimiento a través de diferentes situaciones; que existe un problema en relacionar la teoría con la práctica. El aprendizaje es visto, de modo más realista, como una actividad y el conocimiento como un aspecto de esa actividad y en consecuencia, no simplemente abstraído de él.

La idea es reconocer que el aprendizaje debe estar situado, en el sentido de que el estudiante está localizado en una situación y que lo que es conocido de la experiencia, lo es en relación con un determinado contexto; por lo tanto el conocimiento tiene un carácter contextualizado, lo que significa que no se puede separar el conocimiento a ser aprendido, de las situaciones en la cual es utilizado. ${ }^{10}$

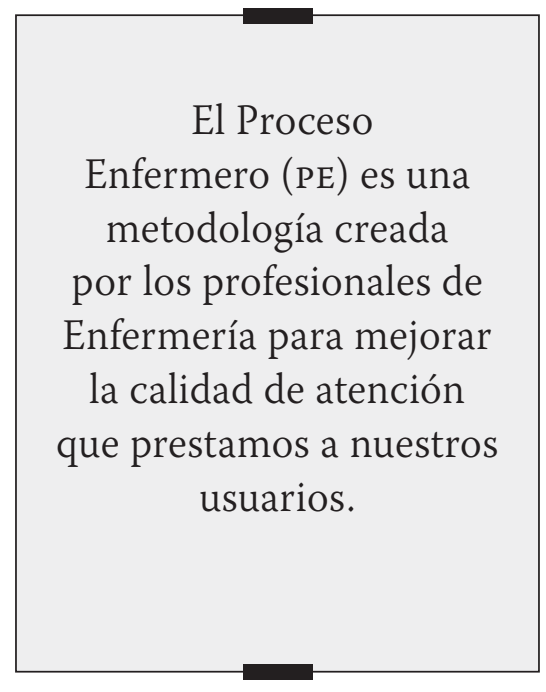

En el caso de enfermería, es una disciplina que requiere que sus practicantes desarrollen herramientas básicas antes de encontrarse con la persona a la que entregará los cuidados, dados los constantes cambios del conocimiento de enfermería han conducido a la búsqueda de elementos que permiten comprender y ampliar el acervo de 
conocimientos para reconocer, integrar e interpretar correctamente cada signo o cada estigma clínico, y en consecuencia aprender a establecer los diagnósticos de enfermería, proporcionar los cuidados oportunos y evaluar la efectividad de los cuidados brindados mediante el uso del NoC.

El Proceso Enfermero (PE) es una metodología creada por los profesionales de Enfermería para mejorar la calidad de atención que prestamos a nuestros usuarios. Este método de trabajo ha sufrido diferentes cambios desde sus inicios, modificaciones que han beneficiado a los enfermeros al lograr la vinculación del paciente como ente participativo de cuidado en los niveles de promoción, prevención, tratamiento y rehabilitación, para que el individuo adquiera el equilibrio entre mente y cuerpo que se traduce en salud. También el PE ha proporcionado ganancia profesional, ya que trabajar con un método estructurado posibilita la unificación de criterios y nos encamina hacia el desarrollo y crecimiento de nuestra profesión. ${ }^{11}$

Dentro de la situación actual del PE en México existen diversos problemas, como son la falta de homogeneidad en las escuelas de enfermería, se ve de manera compleja y diferente a lo largo del plan de estudios, la enfermera clínica no utiliza con frecuencia el PE en el servicio y la investigación sobre la calidad de los cuidados enfermeros está en una etapa inicial. ${ }^{12}$

Esto último se debe a que la etapa del proceso donde menos se hace énfasis es la etapa de evaluación, además de que se sirve de la utilización de la taxonomía NOC la cual se considera la menos conoci- da y quizá las más compleja de llegar a comprender su uso práctico. ${ }^{13}$

El propósito central de esta investigación es identificar cuáles son los factores situacionales que influyen en el aprendizaje de la taxonomía NOC.

\section{METODOLOGÍA}

Se trata de un estudio transversal y correlacional, llevado a cabo de enero a junio de 2010, en alumnos regulares cursando el tercer y cuarto año de la carrera de licenciatura en enfermería, inscritos en el ciclo escolar 2009-2010, siendo estos los criterios de selección en los participantes.

\section{Conceptualización de Variables}

Variables Independientes: Habilidades Docentes, Conocimientos Docentes, Relaciones Interpersonales, Conocimientos Previos, Tiempo de Enseñanza, Evaluación de los Aprendizajes y Retroalimentación de los Aprendizajes.

Variable Dependiente: Aprendizaje de la Taxonomía NOC en los alumnos de enfermería.

El total de alumnos fue de 498, se seleccionó un muestreo estratificado por conglomerados con un grado de confianza de $95 \%$ y un grado de error de $0.01 \leq \varepsilon \leq 0.05$, $0.1 \leq \mathrm{q} \leq 0.2$. Con un total de 224 alumnos de los cuales el $54.5 \%$ son alumnos del cuarto año y el $45.5 \%$ son alumnos del tercer año de la carrera.

El 39.7\% de la población tiene un promedio de calificación de 8.18.5 , el $29.9 \%$ se ubica en el rango de $8.6-9.0$ y el $21.4 \%$ tiene un promedio entre 7.6-8.0. Predomina el sexo femenino con un $83.5 \%$ a los cuales se les aplicó un instrumento modificado a partir del elaborado por Crespo ${ }^{14}$, el cual obtuvo un alfa de Cronbach de 0.975 en el estudio realizado por la autora. El instrumento modificado está constituido por 53 ítems, que exploran 7 dominios: Habilidades Docentes, Conocimientos Docentes, Relaciones Interpersonales ${ }^{15}$, Conocimientos Previos, Tiempo de Enseñanza, Evaluación de los Aprendizajes y Retroalimentación de los Aprendizajes, el cual obtuvo un alfa de Cronbach de 0.984. Lo que permitió identificar la relación que existe entre los factores situacionales y el aprendizaje de la Taxonomía NOC.

El tratamiento estadístico se llevó a cabo mediante la generación de la base de datos correspondiente, en el paquete estadístico SPSS 17.0, se aplicaron métodos estadísticos como son: medidas de tendencia central, se establecieron comparaciones entre variables mediante la prueba de ANOVA, y la prueba t de Student; así como asociación de variables mediante las pruebas Correlación de Pearson y regresión lineal simple. Además de un análisis de componentes principales mediante un análisis factorial.

\section{Aspectos Éticos y Legales.}

El presente trabajo de investigación, no daña o afecta a los participantes de ninguna manera, dentro los estatutos que marca el Reglamento de la Ley General de Salud en Materia de Investigación para la Salud en México, esta investigación se clasifica como investigación sin riesgo por lo tanto está exento de lo obtención del consentimiento informado por escrito, sin embargo, garantiza la confidencialidad en el manejo de la información recabada, así como la identidad de los participantes, los cuales fueron informa- 
dos de los objetivos y características del presente trabajo, y otorgaron su aprobación para la participación en el estudio de manera voluntaria mediante su consentimiento verbal.

\section{RESULTADOS}

Para realizar la interpretación de las medidas de tendencia central, se estableció un rango potencial $(\mathrm{N})^{16}$ (gráfico 1), para lo cual se determinó el promedio de los ítems que componen cada uno de los dominios de la investigación; se determinó la moda, media y mediana, así como la desviación estándar, utilizando la puntuación máxima y mínima; y así establecer el rango.

Al establecer el Coeficiente de Correlación de Pearson entre los diversos dominios antes mencionados se logró un $r_{P}=.908$ con un grado sig. 0.000 entre las dimensiones Evaluación / Retroalimentación lo cual indica que existe una correlación positiva muy fuerte entre estas dos dimensiones del estudio. Esto se verificó al realizar un análisis de regresión lineal simple (gráfico 2); además, con base en el coeficiente de correlación de Pearson se alcanzó el coeficiente de determinación y el resultado de éste nos permite establecer la Varianza de Factores Comunes entre estas dimensiones (cuadro 1).

\section{Gráfico 1. Rango Potencial (\%) - Variable Principal/Dominio}

\section{Adecuado $\square$ Regular $\square$ Inadecuado}

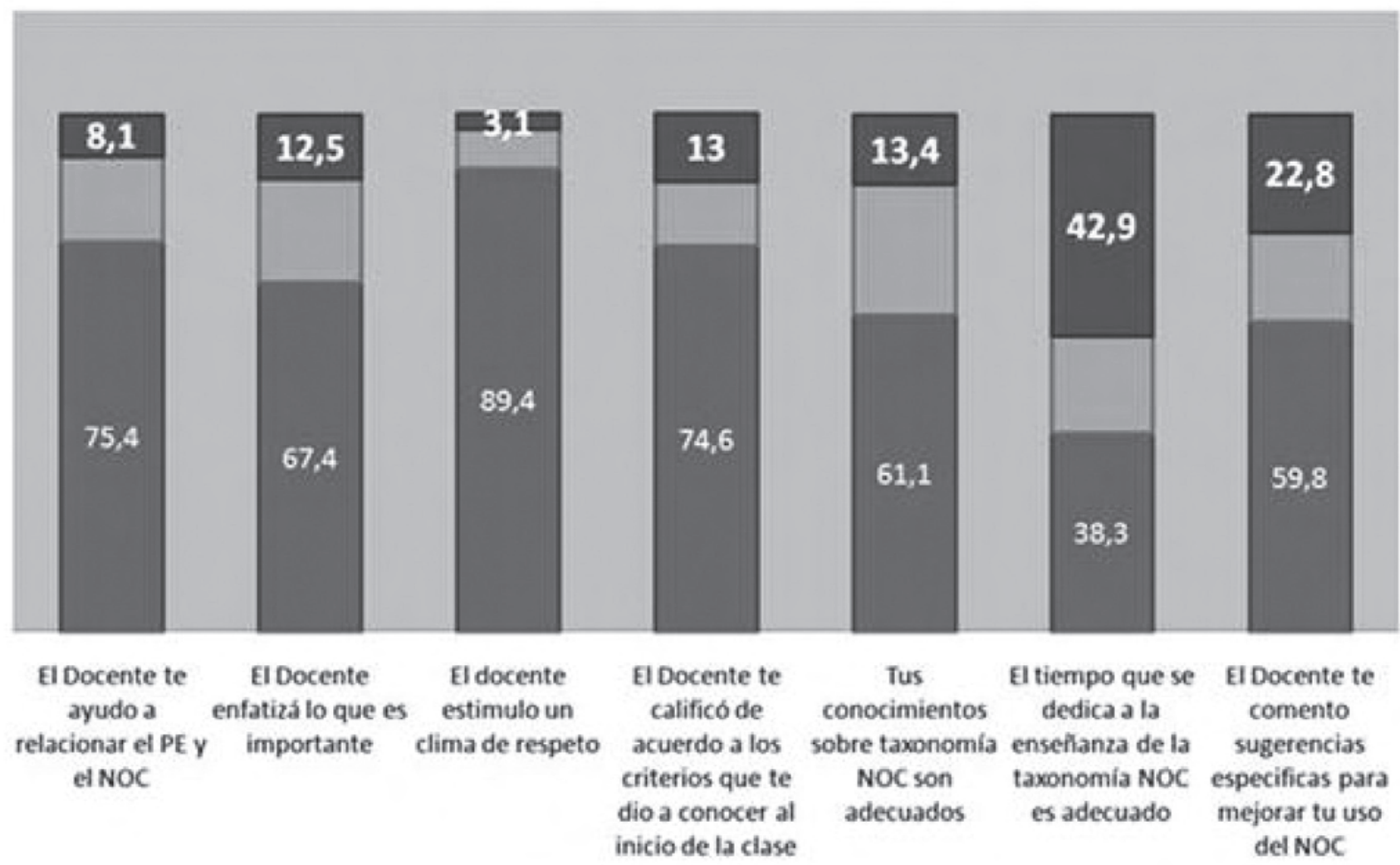

Para interpretar las medidas de tendencia central y la variabilidad, se realizó en su conjunto, mediante la escala de rango potencial, de la cual se interpreto la variable principal de cada dominio del estudio.

En el dominio referente al contexto en el aula la variable "relaciones interpersonales" representado por el ítem: el docente estimulo un clima de respeto, el rango potencial se situó en el $89.4 \%$ como adecuado, siendo el más alto del estudio. En segundo lugar el dominio referente al docente en su variable "habilidades docentes para la enseñanza" representado por el ítem: el docente te ayudo a relacionar el PE y el NOC, obtuvo un
75.4\% como adecuado y finalmente la variable tiempo dedicado a la enseñanza de la taxonomía, correspondiente al dominio del contexto en el aula, representado por el ítem: el tiempo que se dedica a la enseñanza de la taxonomía NOC, sólo el 38.3\% lo calificó como adecuado, siendo el más bajo del estudio. 
Gráfico 2. Varianza dependiente: Evaluación/Retroalimentación

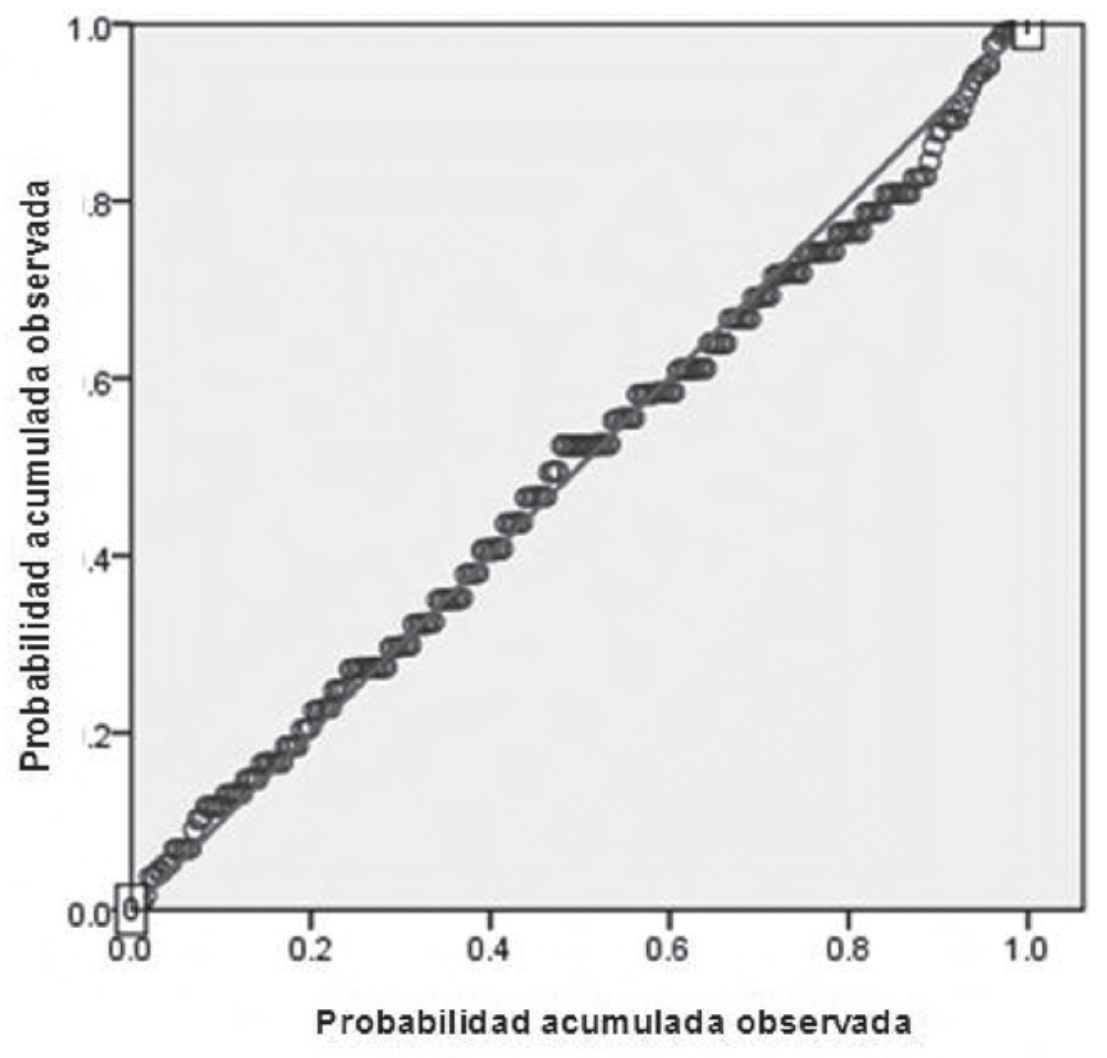

El diagrama de dispersión obtenido, nos permite relacionar las puntuaciones de ambas variables (evaluación de los aprendizajes y retroalimentación), y de esta manera visualizar gráficamente la correlación existente entre ambas, en la cual podemos apreciar una tendencia ascendente, con una dispersión que tiende hacia el centro, es decir, existe una correlación positiva muy fuerte.

Cuadro 1. Varianza de Factores Comunes: Evaluación/Retroalimentación

\begin{tabular}{|c|c|c|c|c|}
\hline \multicolumn{5}{|c|}{ Resumen del modelo b } \\
\hline Modelo & $\mathrm{R}$ & R cuadrado & $\begin{array}{c}\text { R cuadrado } \\
\text { corregida }\end{array}$ & $\begin{array}{c}\text { Error tip. de la } \\
\text { estimación }\end{array}$ \\
\hline 1 & $908^{3}$ & .825 & 824 & 2,26388 \\
\hline \multicolumn{5}{|c|}{ a. Variables predictoras: (Constante), Retroalimentación } \\
\hline
\end{tabular}

Al elevar el coeficiente de $r$ de Pearson se eleva al cuadrado $\left(r^{2}\right)$, se obtiene el coeficiente de determinación lo que indica la varianza de factores comunes, esto es, el porcentaje de la variación de una variable debido a la variación de otra variable; es decir, para la variable "Evaluación" la r es 0,908 y la $r^{2}$ es 0,825, consecuentemente la variable "evaluación de los aprendizajes" explica el 82,5\% de la variación en la variable "retroalimentación" y viceversa. 
En cuanto la correlación que existe entre los factores situacionales y el aprendizaje de la taxonomía NOC se obtuvo:

Correspondientes al Docente la variable Habilidades Docentes, en la correlación de Pearson presentó un $r_{\mathrm{P}}=0.939$ y un grado sig. 0.000 por lo cual se puede decir que tiene una relación positiva muy fuerte. En el caso de la variable Conocimientos Docentes, obtuvo una rP $=0.850$ y una sig. 0.000, es decir, tiene una relación positiva considerable. Por lo cual se acepta la hipótesis de investigación.

En cuanto a los dominios correspondientes del Contexto en el Aula la variable Relaciones Interpersonales refirió un $\mathrm{r}_{\mathrm{P}}=0.748$ con un grado de sig. 0.000 , lo cual significa que tiene una relación positiva media. En el caso de la variable Evaluación de los aprendizajes el resultado fue una $r_{p}=0.888$ con un grado de sig. 0.000 , es decir, existe una relación positiva considerable. La variable Tiempo de Enseñanza tuvo un valor de rP $=0.612$, con una sig. 0.000 , por lo que se puede decir que existe una relación positiva media. Para la variable Retroalimentación, el valor obtenido fue de $r_{P}=0.891$ con un grado de sig. 0.000 , es decir, existe una relación positiva considerable. En el caso de los factores situacionales referentes al contexto en el aula, también se acepta la hipótesis de investigación, al determinar que todas las variables del estudio presentan una relación positiva media y considerable.

Referente al dominio relacionado con el Alumno la variable estudiada Conocimientos Previos, el valor que fue de $r_{P}=0.673$ con un grado sig. 0.000 , lo cual, nos per- mite decir que existe una relación positiva media. Por lo cual podemos decir que se acepta la hipótesis de investigación.

\section{DISCUSIÓN}

En el estudio realizado por Crespo, en el cual se determina que las "Habilidades Docentes" es la variable de mayor importancia en la correlación con el aprendizaje y en segundo término la variable "Relaciones Interpersonales", son las determinantes para establecer el ambiente favorable para el aprendizaje. En el caso de este estudio concuerda en que las "Habilidades Docentes" son el principal elemento para llevar a cabo un aprendizaje de mayor trascendencia para el alumno.

En el caso de la variable "Relaciones Interpersonales", en el estudio realizado por Valongo Zani, Adriana y Suely Noriega, María ${ }^{17}$ en el cual se centra en las relaciones interpersonales como primordiales para el aprendizaje determinando que las de mayor jerarquía son las que existen entre el docente y el alumno, y en segundo lugar, las que existen entre el alumno-alumno; no se concuerda, debido a que sin lugar a dudas, las relaciones interpersonales juegan un papel importante en el aprendizaje en el caso del presente estudio, se situaron en punto intermedio en relación con las demás variables, por lo cual juega un papel secundario en el aprendizaje significativo de la taxonomía NOC.

En el estudio realizado por Cestari, M. E y Loureiro, M;18 se identificó en primer lugar a la variable "Relaciones Interpersonales", y en segundo lugar la variable "Experiencias del alumno" (conocimientos previos), el cual, en este estudio forma parte del aprendizaje en un papel secundario, ya que de las siete variables analizadas, el "conocimiento previo" obtuvo el sexto lugar.

En el caso del presente estudio se observó en segundo lugar la variable "Retroalimentación" y en tercer lugar a la variable "Evaluación", las cuales corresponden a los factores situacionales referentes al contexto en el aula, al igual que las relaciones interpersonales, por lo cual no se descarta a ésta ultima como un elemento básico, para fomentar un ambiente propicio para el aprendizaje.

\section{CONCLUSIONES}

Por lo que se concluye que todos los dominios que componen el estudio presentaron correlación positiva en diferentes grados con respecto al aprendizaje del alumno, obtuvieron mayor importancia los dominios relacionados al docente y fue las "Habilidades Docentes para la Enseñanza" el que más influye para el aprendizaje de la taxonomía NOC, en segundo lugar los dominios relacionados con el Contexto en el Aula: "Retroalimentación de los Aprendizajes" y "Evaluación en Clase"; por lo cual al combinar las habilidades de los docentes para impartir su clase y los elementos de reforzamiento del aprendizaje como son la retroalimentación y la evaluación en clase se pueda incrementar la significancia de los contenidos, para los alumnos de enfermería.

Por último es importante reforzar los conocimientos que tienen los alumnos de enfermería de los elementos teóricos y metodológicos, como son el uso de las taxonomías en especial el Nursing Outcomes Classification (NOC) y el Proceso Enfermero. 


\section{Referencias Bibliográficas}

1 García Alvarado C. Variables Situacionales que Influyen en el Aprendizaje. En: El Paradigma de la Educación Centrada en el Aprendizaje [sede web]. El Triángulo Didáctico [consultado 10 de febrero de 2010]. Disponible en: http://www.carlosgarciaalvarado. com/conalep/Teoria/TeoriaECA4. htm

2 Díaz Barriga F, Hernández G. Estrategias Docentes para un Aprendizaje Significativo. Una Interpretación Constructivista. $2^{\text {a }}$ ed. México: McGraw Hill; 2002.

3 Díaz Barriga F, Hernández G. Constructivismo y Aprendizaje Significativo. Estrategias Docentes para un Aprendizaje Significativo. México: McGraw Hill; 1999. P. 1319.

4 Ausubel D. Psicología Educativa. México: Trillas, 1978. p.769.

5 Ballester A. El aprendizaje significativo en la práctica. Como hacer el aprendizaje significativo en el aula. España: PM 2002. p. 192

6 Díaz Barriga F. Cognición situada y estrategias para el aprendizaje significativo. Revista electrónica de investigación Educativa, 5 (2). 2003. [consultado 2, febrero, 2010]. Disponible en: http://redie. ens.uabc.mx/vol5no2/contenidoarceo.html

7 Chavero Blanco JC. Hipermedia en educación. El modo escritor como catalizador del proceso enseñanza-aprendizaje en la Enseñanza Secundaria Obligatoria, [Tesis Doctoral], Universidad de Extremadura, España; 1998. [consultado 18, febrero, 2010]. Disponible en: http://dialnet.unirioja.es/ servlet/tesis? codigo $=10776$

8 Coll C, et al. El constructivismo en el aula. 18a ed. España: Graó; 2007.

9 García C. El Paradigma de la Educación Centrada en el Aprendizaje. [consultado 28, marzo, 2010]. Disponible en : http://www.carlosgarciaalvarado.com/conalep/Teoria/ TeoriaECA4.htm\#Profesor

10 Laurillard D. La enseñanza como mediación del aprendizaje: El aprendizaje situado. London: Rethinking University Teaching Routledge. 1993. p. 161-290.

11 Díaz Zamora W, Nieto Galvis C. Utilización del proceso de Enfermería en el ejercicio Clínico profesional en seis instituciones prestadoras de servicios de salud. Santafé, Bogotá. 1995. [consultado 10, febrero, 2010]. Disponible en: http://www.encolombia.com/ medicina/enfermeria/enfermeria3200-utilizacion.htm

12 Crespo Knopfler S. Importancia del Proceso Enfermero en el presente y futuro de la profesión. Congreso Nacional de Enfermería Cardiológica. Huatulco, México. 2010. p. 10 -15.

13 Taller de Taxonomías, Taxonomía NOC. [consulta 4, febrero, 2010] Disponible en: http://www.taxo- nomiaenfermera.com/pag/noc. html.

14 Crespo Knopfler S. Factores que inciden en el aprendizaje clínico de los alumnos en el área de ciencias de la salud. [Tesis Doctoral]. Universidad Anáhuac. México. 2008. p. 420

15 Morgan J, Knox J. Characteristics of "best" and "worst" clinical teachers as perceived by university nursing faculty and students. Journal Advanced Nursing 12(3). 1987 p. 331-337. [consultado 2 febrero de 2010]. Disponible en: http://www.ncbi.nlm.nih.gov/pubmed/3648076

16 Hernández Sampieri R, Fernández-Collado C, Baptista Lucio P. Metodología de la Investigación. 4a edición. México: McGraw Hill; 2006. P. 429,430.

17 Valongo Zani A, Suely Nogueira M. Incidentes Críticos del Proceso Enseñanza-Aprendizaje del Curso de Pregrado en Enfermería según la Percepción de los Alumnos y Docentes. Rev. Latino-am Enfermagem 2006; 14(5): 47-54.

18 Cestari ME, Loureiro M. El proceso de enseñanza-aprendizaje en enfermería. Rev Enfermería Global 2005; 7(2). [consultado 30 de enero de 2010]. Disponible en: http://revistas.um.es/eglobal/article/viewFile/468/451 